PROCEEDINGS OF THE

AMERICAN MATHEMATICAL SOCIETY

Volume 131, Number 1, Pages 303-307

S 0002-9939(02)06670-4

Article electronically published on June 12, 2002

\title{
EXISTENCE OF ALGEBRAIC MINIMAL SURFACES FOR AN ARBITRARY PUNCTURE SET
}

\author{
KATSUHIRO MORIYA
}

(Communicated by Bennett Chow)

\begin{abstract}
We will show that any punctured Riemann surface can be conformally immersed into a Euclidean 3-space as a branched complete minimal surface of finite total curvature called an algebraic minimal surface.
\end{abstract}

\section{INTRODUCTION}

The purpose of this paper is to discuss the puncture set problem for algebraic minimal surfaces. The account of the problem is given in the book 3 in the case of unbranched algebraic minimal surfaces.

An algebraic minimal surface in $\mathbb{R}^{3}$ is a branched complete conformal minimal immersion $X: M \rightarrow \mathbb{R}^{3}$ of finite total curvature. In this case, we can consider $M$ as a compact Riemann surface $\bar{M}$ with finitely many points, called puncture points, removed. We will call the set of distinct points $\Sigma$ on $\bar{M}$ such that there exists an algebraic minimal surface $X: \bar{M} \backslash \Sigma \rightarrow \mathbb{R}^{3}$ the puncture set of $\bar{M}$.

We will state the Puncture Set Problem as follows:

The Puncture Set Problem. Given a compact Riemann surface $\bar{M}$, determine the set of puncture sets on $\bar{M}$.

We have known some results on the Puncture Set Problem. Let $\bar{M}_{\ell}$ be a compact Riemann surface of genus $\ell$ and $\operatorname{PS}_{0}^{r}\left(\bar{M}_{\ell}\right)$ the set of $r$ distinct points $\Sigma_{r}$ on $\bar{M}_{\ell}$ such that there exists an unbranched algebraic minimal surface $X: \bar{M}_{\ell} \backslash \Sigma_{r} \rightarrow$ $\mathbb{R}^{3}$. Let $\mathcal{S}\left(\bar{M}_{\ell} ; n, m, m^{\prime}\right)=\left\{\left(p_{1}, \ldots, p_{n}, q_{1}, \ldots, q_{m^{\prime}}\right)\right\}$ be the set of $n+m^{\prime}$ distinct points on $\bar{M}_{\ell}$ such that $\left\{p_{1}, \ldots, p_{n}\right\}=\operatorname{supp}(F)_{-},\left\{q_{1}, \ldots, q_{m}\right\}=\operatorname{supp}(d F)_{+}$and $\left\{q_{1}, \ldots, q_{m}, \ldots q_{m^{\prime}}\right\}=\operatorname{supp}(G)_{+}$, where $F$ and $G$ are meromorphic functions on $\bar{M}_{\ell}$ such that $(G)_{+} \geq(d F)_{+}$. Then

- The set $\operatorname{PS}_{0}^{r}\left(\mathbb{C} P^{1}\right)$ consists of all $r$ distinct points on $\mathbb{C} P^{1}$ ([3, pp. 95-100]).

- The set $\mathcal{S}\left(\bar{M}_{\ell} ; n, m, m^{\prime}\right)$ is contained in $\operatorname{PS}_{0}^{n+m^{\prime}}\left(\bar{M}_{\ell}\right)$ for any $\ell \geq 1$ ([3. p. 127 , Proposition 25 and Proposition 26]).

We will consider branched algebraic minimal surfaces, too. Let $\operatorname{PS}^{r}\left(\bar{M}_{\ell}\right)$ be the set of puncture sets consisting of $r$ distinct points on $\bar{M}_{\ell}$. Then, we will prove the following theorem:

Theorem 1.1. The set $\operatorname{PS}^{r}\left(\bar{M}_{\ell}\right)$ consists of all $r$ distinct points on $\bar{M}_{\ell}$.

Received by the editors February 17, 2000 and, in revised form, August 16, 2001.

2000 Mathematics Subject Classification. Primary 53A10.

Key words and phrases. Minimal surface, Riemann surface, puncture set.

(C)2002 American Mathematical Society 
In other words, any punctured Riemann surface can be conformally immersed into $\mathbb{R}^{3}$ as an algebraic minimal surface.

The author would like to thank R. Miyaoka for her comments on the previous version of this paper.

\section{Preliminaries}

In this section, we will recall the Weierstrass representation formula for minimal surfaces. For more details, see [3].

Let $\bar{M}$ be a compact Riemann surface. For a divisor $D$ on $\bar{M}$, we will denote by mult $_{p} D$ the multiplicity of $D$ at $p, \operatorname{deg} D$ the degree of $D, \operatorname{supp} D$ the support of $D$ and $D_{+}$and $D_{-}$the effective divisors such that $D=D_{+}-D_{-}$:

$$
\begin{gathered}
D=\sum_{p \in \bar{M}}\left(\operatorname{mult}_{p} D\right) \cdot p, \operatorname{deg} D=\sum_{p \in \bar{M}} \operatorname{mult}_{p} D, \\
\operatorname{supp} D=\left\{p \in \bar{M} \mid \operatorname{mult}_{p} D \neq 0\right\}, \\
D_{+}=\sum_{p \in \bar{M}} \max \left\{\operatorname{mult}_{p} D, 0\right\} \cdot p, D_{-}=\sum_{p \in \bar{M}} \max \left\{-\operatorname{mult}_{p} D, 0\right\} \cdot p .
\end{gathered}
$$

For a meromorphic function $g$ on $\bar{M}$ and a meromorphic one-form $\omega$ on $\bar{M}$, let $\operatorname{ord}_{p} g$ and $\operatorname{ord}_{p} \omega$ be the order of $g$ at $p$ and the order of $\omega$ at $p$, respectively. Then, the divisor $(g)$ of $g$ and the divisor $(\omega)$ of $\omega$ are defined by

$$
(g):=\sum_{p \in \bar{M}}\left(\operatorname{ord}_{p} g\right) \cdot p, \quad(\omega):=\sum_{p \in \bar{M}}\left(\operatorname{ord}_{p} \omega\right) \cdot p,
$$

respectively.

Let $M$ be an open Riemann surface, $X: M \rightarrow \mathbb{R}^{3}$ a branched complete conformal minimal immersion and $\Phi_{i}$ the holomorphic one-form on $M$ defined by $\Phi_{i}=\left(\partial X_{i} / \partial z\right) d z$, where $z$ is a local holomorphic coordinate of $M(i=1,2,3)$. The triplet $\Phi=\left(\Phi_{1}, \Phi_{2}, \Phi_{3}\right)$ satisfies the following relation:

$$
\left(\Phi_{1} \otimes \Phi_{1}\right)+\left(\Phi_{2} \otimes \Phi_{2}\right)+\left(\Phi_{3} \otimes \Phi_{3}\right)=0 .
$$

We will call the holomorphic map $[\Phi]: M \rightarrow \mathbb{C} P^{2}$ defined by $[\Phi]:=\left[\Phi_{1}, \Phi_{2}, \Phi_{3}\right]$ in homogenous coordinates on $\mathbb{C} P^{2}$ the generalized Gauss map of $X$. The generalized Gauss map is called an algebraic Gauss map if $M$ is biholomorphic to a compact Riemann surface with a finite set of points removed and $[\Phi]$ extends holomorphically on $\bar{M}$. The removed points are called the puncture points of $X$.

Definition 2.1. We will call a branched complete conformal minimal immersion $X: M \rightarrow \mathbb{R}^{3}$ an algebraic minimal surface if the generalized Gauss map is algebraic.

We can see that the following lemma holds ([1]).

Lemma 2.2. Let $X: M \rightarrow \mathbb{R}^{3}$ be a branched complete conformal minimal immersion. Then, the total curvature of $X$ is finite if and only if the generalized Gauss map is algebraic.

From the above lemma, we can see the following:

Corollary 2.3. Let $X: M \rightarrow \mathbb{R}^{3}$ be a branched complete conformal minimal immersion. Then, $X$ is an algebraic minimal surface if and only if the holomorphic one-form $\Phi_{i}$ on $M$ extends meromorphically on a compact Riemann surface $\bar{M}$ $(i=1,2,3)$. 
In the following, we will assume that $X$ is an algebraic minimal surface and that $M$ is biholomorphic to a compact Riemann surface $\bar{M}_{\ell}$ of genus $\ell$ with puncture points $\left\{p_{1}, \ldots, p_{r}\right\}$ removed.

Definition 2.4. For $\Phi=\left(\Phi_{1}, \Phi_{2}, \Phi_{3}\right)$, we will define the divisor $(\Phi)$ of $\Phi$ by

$$
(\Phi):=\sum_{p \in \bar{M}_{\ell}}\left(\min _{i=1,2,3} \operatorname{ord}_{p} \Phi_{i}\right) \cdot p .
$$

Then, we can see that $\operatorname{supp}(\Phi)_{+}$is the set of all the branch points of $X$ and $\operatorname{supp}(\Phi)_{-}$is the set of all the puncture points of $X$. From the completeness of $X$, the multiplicity of $(\Phi)_{-}$at each puncture point is equal to or greater than 2 :

$$
\operatorname{supp}(\Phi)_{-}=\left\{p_{1}, \ldots, p_{r}\right\}, \operatorname{mult}_{p_{i}}(\Phi)_{-} \geq 2(i=1, \ldots, r) .
$$

Conversely, from a triplet $\Phi$ of meromorphic one-forms on $\bar{M}_{\ell}$ satisfying the condition (2.1) and (2.2), we can obtain a multi-valued algebraic minimal surface $X: M \rightarrow \mathbb{R}^{3}$ by integration

$$
X(p):=\operatorname{Re} \int_{p_{0}}^{p} \Phi
$$

where $p$ and $p_{0}$ are non-puncture points.

Definition 2.5. We will call the condition (2.2) the divisor condition.

If we choose another base point in the integral, then the image of the map shifts by a translation in $\mathbb{R}^{3}$.

We can see that the multi-valued algebraic minimal surface $X$ can be reduced to a single-valued algebraic minimal surface if and only if

$$
\operatorname{Re} \int_{\gamma_{i}} \Phi=(0,0,0)(i=1, \ldots, 2 \ell+r-1),
$$

where $\gamma=\left\{\gamma_{1}, \ldots, \gamma_{2 \ell+r-1}\right\}$ is a basis of the first homology group $H_{1}(M, \mathbb{Z})$ of $M$.

Definition 2.6. We will call the condition (2.4) the period condition.

We can obtain a pair $(g, \omega)$ consisting of a meromorphic function $g$ on $\bar{M}_{\ell}$ and a meromorphic one-form $\omega$ on $\bar{M}_{\ell}$ which is equivalent to $\Phi$ by

$$
\begin{gathered}
(g, \omega)=\left(\frac{\Phi_{3}}{\Phi_{1}-\sqrt{-1} \Phi_{2}}, \Phi_{1}-\sqrt{-1} \Phi_{2}\right), \\
\left(\Phi_{1}, \Phi_{2}, \Phi_{3}\right)=\left(1-g^{2}, \sqrt{-1}\left(1+g^{2}\right), 2 g\right) \frac{\omega}{2} .
\end{gathered}
$$

When the triplet $\Phi$ corresponds to a single-valued minimal surface $X: M \rightarrow \mathbb{R}^{3}$ by (2.3), the pair $(g, \omega)$ is called the Weierstrass data of $X$. It is well-known that $g$ is the stereographic projection of the Gauss map of $X$ from the north pole.

We can see that the following relation holds among $(g),(\omega)$, and $(\Phi)$ :

$$
(\Phi)=-2(g)_{-}+(\omega) .
$$

Then, the divisor condition (2.2) becomes as follows:

$$
\begin{gathered}
\operatorname{supp}\left(-2(g)_{-}+(\omega)\right)_{-}=\left\{p_{1}, \ldots, p_{r}\right\}, \\
\operatorname{mult}_{p_{i}}\left(-2(g)_{-}+(\omega)\right)_{-} \geq 2(i=1, \ldots, r) .
\end{gathered}
$$




\section{Proof of Theorem 1.1]}

In this section, we will prove Theorem 1.1

Let $\operatorname{Div}\left(\bar{M}_{\ell}\right)$ be the set of divisors on $\bar{M}_{\ell}$ and $\operatorname{Div}_{+}^{d}\left(\bar{M}_{\ell}\right)=\left\{D \in \operatorname{Div}\left(\bar{M}_{\ell}\right) \mid D>\right.$ $0, \operatorname{deg} D=d\}$. For a divisor $D \in \operatorname{Div}\left(\bar{M}_{\ell}\right)$, let $\mathcal{I}(D)$ be the complex vector space of meromorphic one-forms on $\bar{M}_{\ell}$ defined by $\mathcal{I}(D):=\{\xi \mid(\xi) \geq D\}$ and $i(D)$ the complex dimension of $\mathcal{I}(D)$.

We will assume the following lemmas:

Lemma 3.1 ([3, Proposition 3]). Let $\omega$ be a meromorphic one-form on $\bar{M}_{\ell}$. Then, we have $\operatorname{deg}(\omega)=2 \ell-2$.

Lemma $3.2\left(\left[3\right.\right.$, p. 35]). Let $-D \in \operatorname{Div}_{+}^{d}\left(\bar{M}_{\ell}\right)$. Then, we have $i(D)=\ell+d-1$.

Lemma 3.3 ([3, Proposition 10]). If $D \in \operatorname{Div}_{+}^{d}\left(\bar{M}_{\ell}\right), d \geq 2 \ell$, then there exists a meromorphic function $g$ such that $(g)_{-}=D$.

Then, we can prove the following lemmas:

Lemma 3.4. For any distinct points $\left\{p_{1}, \ldots, p_{r}\right\} \subset \bar{M}_{\ell}$, there exists a meromorphic function $g$ on $\bar{M}_{\ell}$ such that $\operatorname{supp}(g)_{-}=\left\{p_{1}, \ldots, p_{r}\right\}$.

Proof. Let $d \geq 2 \ell$ and $D \in \operatorname{Div}_{+}^{d}\left(\bar{M}_{\ell}\right)$ such that $\operatorname{supp} D=\left\{p_{1}, \ldots, p_{r}\right\}$. Then, there exists a meromorphic function $g$ such that $(g)_{-}=D$ by Lemma 3.3 Then, $\operatorname{supp}(g)_{-}=\operatorname{supp} D=\left\{p_{1}, \ldots, p_{r}\right\}$.

Lemma 3.5. Let $-D \in \operatorname{Div}_{+}^{d}\left(\bar{M}_{\ell}\right)$ with supp $D=\left\{p_{1}, \ldots, p_{r}\right\}$. If $g$ is a meromorphic function on $\bar{M}_{\ell}$ such that $\operatorname{supp}(g)_{-}=\left\{p_{1}, \ldots, p_{r}\right\}$ and $d \leq 2\left(\operatorname{mult}_{p_{i}}(g)_{-}-\ell\right)$ $(i=1, \ldots, r)$, then the pair $(g, \omega)$ satisfies the condition 2.5 for any $\omega \in \mathcal{I}(D) \backslash$ $\{0\}$.

Proof. Let $\omega \in \mathcal{I}(D) \backslash\{0\}$. It is clear that if $p \notin\left\{p_{1}, \ldots, p_{r}\right\}$, then

$$
\operatorname{mult}_{p}\left(-2(g)_{-}+(\omega)\right)_{-}=0 \text {. }
$$

If $p \in\left\{p_{1}, \ldots, p_{r}\right\}$, then

$$
\begin{aligned}
\operatorname{mult}_{p}( & \left.-2(g)_{-}+(\omega)\right)_{-} \geq \operatorname{mult}_{p}\left(-2(g)_{-}+(\omega)_{+}\right)_{-} \\
& =\max \left\{\operatorname{mult}_{p}\left(2(g)_{-}-(\omega)_{+}\right), 0\right\} \\
& \geq \max \{(2 \ell+d)-(2 \ell+d-2), 0\}=2,
\end{aligned}
$$

since $\operatorname{deg}(\omega)_{+}=2 \ell-2+\operatorname{deg}(\omega)_{-} \leq 2 \ell+d-2$ by Lemma 3.1 and the assumption of Lemma 3.5. Hence, the pair $(g, \omega)$ satisfies (2.5).

Remark 3.6. From the assumption in Lemma 3.5, we can see that $r \leq d$.

Let $(g, \omega)$ be a pair satisfying $(2.5)$ and let $\mathcal{P}=\left(\mathcal{P}_{1}, \ldots, \mathcal{P}_{3(2 \ell+r-1)}\right): \mathcal{I}(D) \rightarrow$ $\mathbb{R}^{3(2 \ell+r-1)}$ be a real linear map defined by

$$
\begin{gathered}
\mathcal{P}_{i}(\omega)=\operatorname{Re} \int_{\gamma_{i}}\left(1-g^{2}\right) \omega \\
\mathcal{P}_{(2 \ell+r-1)+i}(\omega)=\operatorname{Re} \int_{\gamma_{i}} \sqrt{-1}\left(1+g^{2}\right) \omega, \\
\mathcal{P}_{2(2 \ell+r-1)+i}(\omega)=\operatorname{Re} \int_{\gamma_{i}} g \omega
\end{gathered}
$$

$(i=1, \ldots, 2 \ell+r-1)$, where $\left\{\gamma_{1}, \ldots, \gamma_{2 \ell+r-1}\right\}$ is a basis for $H_{1}(M, \mathbb{Z})$. We will denote by $\operatorname{ker} \mathcal{P}$ the kernel of $\mathcal{P}$ and $\operatorname{dim}_{\mathbb{R}} \operatorname{ker} \mathcal{P}$ the real dimension of ker $\mathcal{P}$. 
Lemma 3.7. If $2 d>4 \ell+3 r-1$, then $\operatorname{dim}_{\mathbb{R}} \operatorname{ker} \mathcal{P}>0$.

Proof. By Lemma 3.2 the real dimension of $\mathcal{I}(D)$ is equal to $2(\ell+d-1)$. Hence, if $2(\ell+d-1)>3(2 \ell+r-1)$, that is, $2 d>4 \ell+3 r-1$, then $\operatorname{dim}_{\mathbb{R}} \operatorname{ker} \mathcal{P} \neq 0$. Thus, $\operatorname{dim}_{\mathbb{R}} \operatorname{ker} \mathcal{P}>0$.

Proof of Theorem 1.1. Let $\left\{p_{1}, \ldots, p_{r}\right\}$ be a set of distinct points on $\bar{M}_{\ell}$ and $d$ a positive integer such that $d \geq r$ and $2 d>4 \ell+3 r-1$. By Lemma 3.4 we can see that there exists a meromorphic function $g$ such that $\operatorname{supp}(g)_{-}=\left\{p_{1}, \ldots, p_{r}\right\}$. We may assume that 2 mult $_{p_{i}}(g)_{-} \geq 2 \ell+d(i=1, \ldots, r)$. Let $-D \in \operatorname{Div}_{+}^{d}\left(\bar{M}_{\ell}\right)$ such that $\operatorname{supp} D=\left\{p_{1}, \ldots, p_{r}\right\}$. By Lemma 3.5, the pair $(g, \omega)$ satisfies the condition (2.5) for any $\omega \in \mathcal{I}(D) \backslash\{0\}$. We can see that ker $\mathcal{P} \backslash\{0\}$ is a set of Weierstrass data for algebraic minimal surfaces from $\bar{M}_{\ell} \backslash\left\{p_{1}, \ldots, p_{r}\right\}$ into $\mathbb{R}^{3}$. By Lemma 3.7 $\operatorname{dim}_{\mathbb{R}} \operatorname{ker} \mathcal{P}>0$. Hence, $\operatorname{ker} \mathcal{P} \backslash\{0\} \neq \emptyset$.

Remark 3.8. When we consider that algebraic minimal surfaces are similar to meromorphic functions on a compact Riemann surface, we may consider that Theorem 1.1 is similar to Lemma 3.4. We will discuss deformations of unbranched algebraic minimal surfaces from this point of view in [2].

\section{REFERENCES}

1. K. Moriya, On a variety of algebraic minimal surfaces in Euclidean 4-space, Tokyo J. Math. 21 (1998), no. 1, 121-134. MR 99h:53010

2. _ Deformations of complete minimal surfaces of genus one with one end and finite total curvature, preprint.

3. K. Yang, Complete minimal surfaces of finite total curvature, Kluwer Academic Publishers Group, Dordrecht, 1994. MR 96d:53009

Institute of Mathematics, University of Tsukuba, Tsukuba, Ibaraki, 305-8571, Japan

E-mail address: moriya@math.tsukuba.ac.jp 